\title{
Comparative study of the dynamics of laser and acoustically generated bubbles in viscoelastic media
}

\author{
Chad T. Wilson, ${ }^{1}$ Timothy L. Hall, ${ }^{1}$ Eric Johnsen, ${ }^{2}$ Lauren Mancia, ${ }^{2}$ Mauro Rodriguez, ${ }^{2}$ Jonathan E. Lundt, ${ }^{1}$ Tim Colonius, ${ }^{3}$ \\ David L. Henann, ${ }^{4}$ Christian Franck,${ }^{5}$ Zhen Xu, ${ }^{1, *}$ and Jonathan R. Sukovich ${ }^{1, \dagger}$ \\ ${ }^{1}$ Department of Biomedical Engineering, University of Michigan, Ann Arbor, Michigan 48105, USA \\ ${ }^{2}$ Department of Mechanical Engineering, University of Michigan, Ann Arbor, Michigan 48105, USA \\ ${ }^{3}$ Department of Mechanical Engineering, California Institute of Technology, Pasadena, California, 91125, USA \\ ${ }^{4}$ Department of Mechanical Engineering, Brown University, Providence, Rhode Island 02912, USA \\ ${ }^{5}$ Department of Mechanical Engineering, University of Wisconsin, Madison, Wisconsin 53706, USA
}

(Received 27 August 2018; revised manuscript received 24 January 2019; published 10 April 2019)

\begin{abstract}
Experimental observations of the growth and collapse of acoustically and laser-nucleated single bubbles in water and agarose gels of varying stiffness are presented. The maximum radii of generated bubbles decreased as the stiffness of the media increased for both nucleation modalities, but the maximum radii of laser-nucleated bubbles decreased more rapidly than acoustically nucleated bubbles as the gel stiffness increased. For water and low stiffness gels, the collapse times were well predicted by a Rayleigh cavity, but bubbles collapsed faster than predicted in the higher stiffness gels. The growth and collapse phases occurred symmetrically (in time) about the maximum radius in water but not in gels, where the duration of the growth phase decreased more than the collapse phase as gel stiffness increased. Numerical simulations of the bubble dynamics in viscoelastic media showed varying degrees of success in accurately predicting the observations.
\end{abstract}

DOI: 10.1103/PhysRevE.99.043103

\section{INTRODUCTION}

Cavitation is known to generate complex, often violent, conditions within and surrounding bubbles and has been widely studied for a range of associated phenomena [1-9] and applications [10-13]. Recently, there has been a surge in interest in cavitation in the medical community where, for example, cavitation activity is suspected of contributing to traumatic brain injury associated with exposure to blasts and impacts $[14,15]$. Controlled, acoustically generated cavitation is also being explored for therapeutic/surgical applications using a technique known as histotripsy. Histotripsy utilizes short-duration $(\leqslant 20 \mu \mathrm{s})$, high-amplitude $(P-\geqslant 15 \mathrm{MPa})$ focused acoustic pulses to controllably generate near-vacuum microbubbles in tissues, which act to mechanically fractionate and destroy a wide range of tissues [16-21].

Despite recent medical interests, however, the mechanisms by which cavitation damages tissue remain poorly understood $[22,23]$. The susceptibility to cavitation-induced damage can vary significantly depending on the tissues' mechanical properties [24,25]. Modeling efforts that take into account the viscoelastic properties of tissues and tissue-mimicking hydrogels have shown varying degrees of success in replicating observations $[23,25-27]$. The inability of models to fully replicate observations is due, in part, to the incomplete characterization of materials' viscoelastic properties [28-32], especially when materials are subjected to the high to ultrahigh strain-rates $\left(10^{3}-10^{8} \mathrm{~s}^{-1}\right)$ expected during cavitation. To the best of our knowledge, experimental techniques to directly measure the viscoelastic properties of materials subjected to these types

\footnotetext{
*zhenx@umich.edu

†jsukes@umich.edu
}

of strain-rates do not exist, but observations suggest that they may vary significantly from those measured at quasistatic strain rates $[15,27,33]$.

Experimental investigations of bubble dynamics in aqueous and viscoelastic media have typically relied on two methods, acoustic- and laser-induced cavitation, to nucleate bubbles. While it may be reasonable to expect that acoustically nucleated bubbles would be more representative of cavitation in biological contexts, e.g., during brain injury, a number of challenges have impeded the controlled study of these bubbles in viscoelastic media. In particular, generating single, spherical bubbles at precisely controlled locations using transient acoustic pulses [8,34] is difficult to accomplish without modifying the media, for example, by injecting gas microbubbles or inserting point defects in the media [35].

As such, experimental studies of bubble dynamics in these media have generally been conducted using laser-based mechanisms for nucleation as these methods provide precise temporal and spatial control over cavitation generation [27,36-39]. However, the extent to which the dynamics of laser-generated bubbles represent those of acoustically generated bubbles is unclear owing to the nucleation mechanism, whereby ionization events may locally modify the material properties of the surrounding media and can lead to the generation of excess vapor and gas byproducts within these bubbles which may thus influence collapse outcomes $[37,38,40,41]$. Further, due to the complex physics underlying the formation and growth of laser-nucleated bubbles [42-44], and the broader interest in bubble collapse and rebound events in general, models of the initial growth of bubbles remain underdeveloped.

We are thus motivated to perform a comparative study of the dynamics of both acoustically and laser-generated 


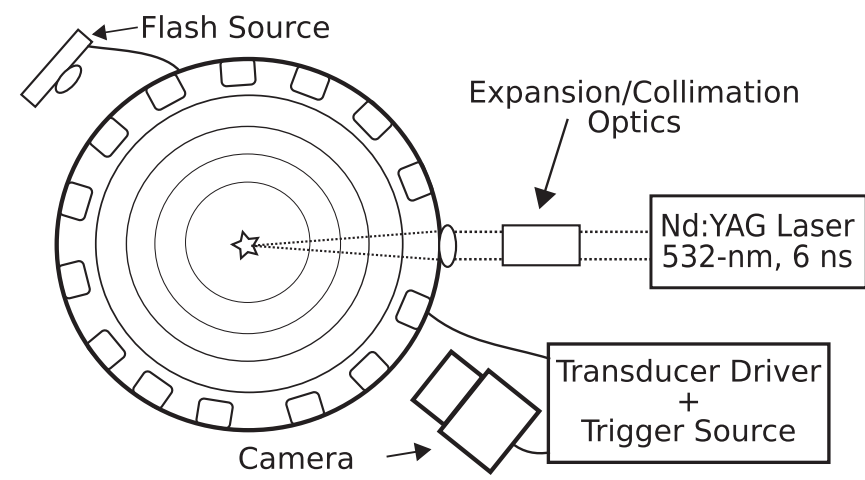

FIG. 1. Schematic drawing of the experimental setup, top-down view. Gel samples were lowered into the transducer from above (into the page).

cavitation bubbles, with the goal of providing insight into how bubble dynamics are affected by the viscoelastic properties of the media, and whether and/or how the dynamics are affected by nucleation mechanism. Experimental observations of the initial growth and first collapse of both acoustically and laser-nucleated single bubbles are presented for water and for tissue-mimicking agarose gels of varying stiffness. Numerical simulations of the bubble dynamics in viscoelastic media are then compared with the observations in order to assess their efficacy in reproducing the experimental results, and to determine the extent to which laser-generated bubbles may serve as surrogates for acoustically nucleated ones.

\section{METHODS}

\section{A. Experimental setup}

Experiments were carried out in a custom-built, 10-cmdiameter, open-topped, spherical acoustic array, populated with 16, 2-cm-diameter, focused transducer elements with a center frequency of $1 \mathrm{MHz}$. Two pairs of 25-mm-diameter optical windows were placed along the equatorial plane of the transducer for illumination and imaging, and a 50-mmdiameter laser access window was included for laser nucleation. A 5.8-cm-diameter opening at the top of the transducer allowed for gel sample insertion. A schematic drawing of the experimental setup is shown in Fig. 1.

During experiments single bubbles were generated at the center of the sphere using two methods, laser and acoustic nucleation. Laser-nucleated bubbles were generated using a pulsed Nd:YAG laser (Continuum, Surelite I), frequency doubled to $532 \mathrm{~nm}$ with a pulse duration of $6 \mathrm{~ns}$. The beam of the laser was expanded to a diameter of $40 \mathrm{~mm}$ and focused to the center of the sphere using a 75-mm focal length lens. Acoustically generated bubbles were nucleated using a 1.5cycle acoustic pulse containing only a single, large rarefactional pressure half-cycle. As both nucleation methods rely on threshold phenomena to generate cavitation, the volume of the field exposed to super-threshold irradiance/pressure capable of generating bubbles increases with the intensity of the generating pulses. Instead of generating larger single bubbles this preferentially generates multiple bubbles in the focal region. To minimize the generation of multiple bubbles, the irradi-
TABLE I. Mechanical properties of media used in this study as given in Ref. [25].

\begin{tabular}{lccc}
\hline \hline Medium & $\begin{array}{c}\text { Young's modulus } \\
(\mathrm{kPa})\end{array}$ & $\begin{array}{c}\text { Density } \\
\left(\mathrm{kg} / \mathrm{m}^{3}\right)\end{array}$ & Water content $(\%)$ \\
\hline Water & - & 998 & 100 \\
0.3\% Agarose & $1.13 \pm 0.47$ & 1003.0 & 98.8 \\
1.0\% Agarose & $21.7 \pm 1.0$ & 1010.0 & 98.1 \\
2.5\% Agarose & $242 \pm 27$ & 1025.0 & 96.7 \\
$5.0 \%$ Agarose & $570 \pm 46$ & 1050.0 & 94.3 \\
\hline \hline
\end{tabular}

ance and rarefactional pressure thresholds for nucleation were therefore determined empirically prior to experiments. This was done by adjusting the respective laser pulse energy and acoustic focal pressure such that the probability of generating cavitation during a given attempt was approximately $50 \%$. For laser nucleation this resulted in a pulse energy of $5 \mathrm{~mJ}$ per pulse, and for acoustic nucleation the rarefactional focal pressure was approximately $-24 \mathrm{MPa}$ [8].

During all experiments the transducer was filled with deionized water, filtered to $2 \mu \mathrm{m}$ and degassed to $4 \mathrm{kPa}$. Bubbles were generated by both nucleation mechanisms in water and agarose gels with concentrations of $0.3 \%, 1.0 \%, 2.5 \%$, and $5.0 \%$ (weight/volume). The gels were prepared following a modified set of the procedures detailed in Ref. [25], where in the present study the gels were allowed to solidify at room temperature $\left(17.8 \pm 0.6^{\circ} \mathrm{C}\right)$ instead of at $4^{\circ} \mathrm{C}$. The impact of this modification on the resultant stiffness values of the gels in the present study compared to those in Ref. [25] is expected to be $\lesssim 5 \%$ [45]. The mechanical properties of the gels as given in Ref. [25] are shown in Table I. It should be noted that the properties of the gels in Ref. [25] were measured under quasistatic loading conditions using a parallel-plate rheometer. Gel samples were prepared in $7.5 \mathrm{~cm}$ long, cylindrical syringes with diameters of $2.5 \mathrm{~cm}$, following which the samples were mounted to a positioning system, submerged in the water through the opening on top of the sphere, and positioned at the focal spot of the laser/acoustic array for nucleation. To ensure that the dynamics of the generated bubbles were not influenced by local defects in the gels, i.e., structural changes due to previously generated cavitation events, only a single nucleation event was generated at each focal site within the gels, and all bubbles were nucleated $\gtrsim 20 R_{m}(\geqslant 5 \mathrm{~mm})$ from any previously generated bubbles in the gel sample. All gel samples had specific acoustic impedances within $<5 \%$ of that of water's and/or were physically large enough to be regarded as infinite with respect to the nucleated bubbles.

Images of the generated bubbles were captured using a high-speed camera (Vision Research, Phantom v2012) at a fixed frame rate of $400 \mathrm{kHz}$. From the time point of nucleation, all image series extended $\geqslant 100 \mu \mathrm{s}$. Images were illuminated using a pulsed, blue LED backlight source with a minimum flash width of $20 \mathrm{~ns}$. To capture the high-speed dynamics associated with growth and collapse, a multi-flashper-camera-exposure imaging technique was used. During exposures near the initial growth and first collapse, the LED backlight was pulsed two to four times per single camera exposure. A schematic drawing of this imaging technique, with example images, is shown in Fig. 2. 


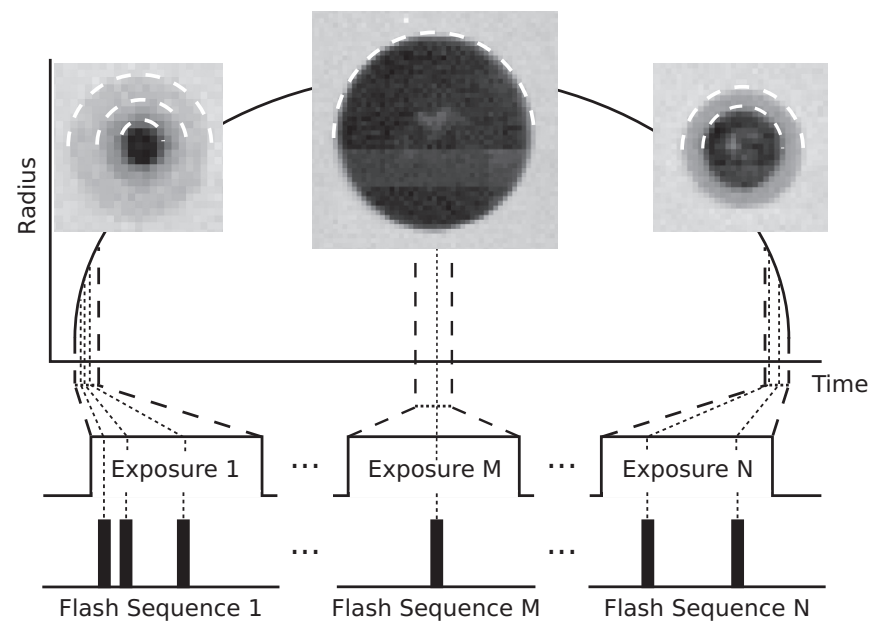

FIG. 2. Visualization of the multiflash imaging technique with timing diagrams of the variable flash sequences used during each phase of the bubble's evolution with example images.

Flash timings could be adjusted independently of the camera exposure and spacings between individual flashes within single image frames ranged from $100 \mathrm{~ns}$ to $1.3 \mu \mathrm{s}$, depending on the experiment. This allowed the growth and collapse events to be acquired with greater temporal resolution than was possible with the native camera settings. This imaging technique resulted in nested, concentric bubbles in images and brightness thresholding and edge detection were used to differentiate them. The radii of the captured bubbles were calculated by least-squares circle fitting to their detected boundaries. Bubbles were rejected for inclusion if the variance in the radii of the individual points detected along the boundary exceeded that which would be expected for fluctuations about the fit radius of more than $5 \%$ in any frame of the image series except those captured within $\leqslant 1 \mu$ s of the nucleation event or collapse point. These frames were excluded from consideration for rejecting bubbles owing to the small size of the bubbles at these time points and/or interference from the laser plasma in images. Using the multiflash imaging technique it was not always possible to reliably determine which detected bubble corresponded to which flash in a given exposure, especially near the collapse point where the bubble wall velocity changes direction. Radii with indeterminate time points were thus excluded from analysis.

\section{B. Theoretical modeling}

The dynamics of a single isolated, spherical, gas-filled bubble in both water and in homogeneous viscoelastic media (representative of the agarose gels) are numerically simulated using an approach similar to previous studies of histotripsy cavitation [25,26,46-48]. In the present study, only acoustically nucleated bubbles are modeled. This is because the physics of laser nucleation is more complex than acoustic nucleation, involving plasma formation and recombination as well as rupture of the gel material adjacent to the nucleation site-developing a new model for the initial growth of lasernucleated bubbles is beyond the scope of the present work.

In the model described in this section, there are two important unknowns: the bubble's initial size and the material
TABLE II. Physical constants used in simulations.

\begin{tabular}{lc}
\hline \hline Parameter & Value \\
\hline$c_{\infty}$ & $1497 \mathrm{~m} / \mathrm{s}$ \\
$\rho_{\infty}$ & $1000 \mathrm{~kg} / \mathrm{m}^{3}$ \\
$p_{\infty}$ & $101.325 \mathrm{kPa}$ \\
$T_{\infty}$ & $25^{\circ} \mathrm{C}$ \\
$S$ & $0.072 \mathrm{~N} / \mathrm{m}$ \\
$\mu_{w}$ & $0.001 \mathrm{~Pa} \cdot \mathrm{s}$ \\
$\mu_{a}$ & $0.115 \mathrm{~Pa} \cdot \mathrm{s}$ \\
$K_{A}$ & $5.28 \times 10^{-5} \mathrm{~W} / \mathrm{mK}^{2}$ \\
$K_{B}$ & $1.165 \times 10^{-2} \mathrm{~W} / \mathrm{mK}$ \\
$\kappa$ & 1.4 \\
$K_{M}$ & $0.55 \mathrm{~W} / \mathrm{mK}$ \\
$C_{p}$ & $4.181 \times 10^{3} \mathrm{~J} / \mathrm{kgK}$ \\
\hline \hline
\end{tabular}

properties of the surroundings. It is not possible to uniquely extract both of these unknowns by fitting to the experimental data. Therefore, for simplicity, we assume that the stiffnesses of the gels measured under quasistatic conditions (Table I) are representative of the values at high strain-rates relevant to bubble dynamics. The reported values of agarose's viscosity vary widely over several orders of magnitude $[23,49]$ and cannot be measured under conditions relevant to the present study using currently available techniques [50]. As such, the viscosity of agarose used in simulations is chosen based on the criterion that it be on the same order of magnitude as values reported previously [23], but is otherwise arbitrary. We acknowledge that these simplifications can contribute to discrepancies between simulations and experiments. A more detailed exploration of the simulation parameter space in relation to the present work will be presented in a future study.

Two sets of simulations are carried out using the specified material properties: one where the gel viscosity is taken to be equal to that of water's $[25,47,51], \mu_{w}$, and another where it is taken to be the prescribed viscosity for agarose, $\mu_{a}$ (Table II). With the material properties specified, we then calibrate the bubble's unknown initial size by comparing with the experimental data.

To account for near-field compressibility effects, radial bubble dynamics are described by the Keller-Miksis equation [52] extended to include elasticity [30]:

$$
\begin{aligned}
(1 & \left.-\frac{\dot{R}}{c_{\infty}}\right) R \ddot{R}+\frac{3}{2}\left(1-\frac{\dot{R}}{3 c_{\infty}}\right) \dot{R}^{2} \\
= & \frac{1}{\rho_{\infty}}\left(1+\frac{\dot{R}}{c_{\infty}}+\frac{R}{c_{\infty}} \frac{d}{d t}\right) \\
& \times\left\{p_{B}-\left[p_{\infty}+p_{f}(t)\right]-\frac{2 S}{R}+J\right\},
\end{aligned}
$$

where $R$ is the time-dependent bubble radius, $c_{\infty}$ and $\rho_{\infty}$ are the constant sound speed and density of the surrounding medium, respectively, $p_{B}$ is the internal bubble pressure, $S$ is the surface tension, and $J$ is the integral of deviatoric stresses in the surroundings [Eq. (3)]. All constants correspond to water or air at a temperature of $25^{\circ} \mathrm{C}$ (Table II) unless otherwise specified.

Acoustic excitation is modeled as a time varying far-field pressure acting on the bubble. The far-field pressure is the 
sum of the ambient pressure, $p_{\infty}$, and time-varying acoustic forcing, $p_{f}(t)$ :

$$
p_{f}(t)= \begin{cases}p_{A}\left(\frac{1+\cos [\omega(t-\delta)]}{2}\right)^{n}, & |t-\delta| \leqslant \frac{\pi}{\omega}, \\ 0, & |t-\delta|>\frac{\pi}{\omega} .\end{cases}
$$

The pressure amplitude, $p_{A}=-24 \mathrm{MPa}$, and frequency, $f=$ $1 \mathrm{MHz}(\omega=2 \pi f)$, correspond to experimental measurements. The time delay, $\delta=5 \mu \mathrm{s}$, and fitting parameter, $n=$ 3.7 , were chosen as in previous studies $[25,26,46,47]$. Here, it is hypothesized that the largest-amplitude cycle of the waveform gives rise to the bubble growth.

Agarose is modeled with a finite-deformation Kelvin-Voigt constitutive equation [30] in which the elastic component of the material response is given through the Neo-Hookean model, resulting in the following integral of the deviatoric contribution of the stresses in the surrounding medium:

$$
J=-\frac{4 \mu \dot{R}}{R}-\frac{E}{6}\left[5-4\left(\frac{R_{0}}{R}\right)-\left(\frac{R_{0}}{R}\right)^{4}\right],
$$

where $\mu$ is the viscosity and $E$ is the ground-state Young's modulus. As previously discussed, simulations use the quasistatic Young's modulus reported for each agarose gel concentration (Table I) and are carried out using the viscosity of water, $\mu=\mu_{w}$, in all media and additionally with the prescribed viscosity of agarose, $\mu=\mu_{a}$, in the gels. In Eq. (3), $R_{0}$ is the bubble radius when the surroundings are stress-free, which, in the present work, we take to represent the initial bubble radius.

Experimentally inferred cavitation nuclei in water are on the order of nanometers [8], but direct measurements are not feasible on this scale. For these simulations, the initial radii of the bubbles in each medium are empirically determined in an iterative fashion by adjusting the initial radii in simulations in order to produce the best agreement between the simulated maximum radii and those measured experimentally from a representative set of cavitation events in each medium. The representative data sets for each medium are defined as those which have maximum radii closest to the mean maximum radius of all bubbles measured in the given medium.

Heat transfer effects are incorporated by solving for temperature fields inside and outside of the bubble following the approaches of Refs. [31,53-55]. These simulations take the bubble wall to be impervious to gas and neglect vapor inside the bubble. While this simplification risks underpredicting the lifespan of the bubble and neglects subsequent rebounds [56], this error is expected to be minor when considering the single cycle of bubble growth and collapse presented herein. The time derivative of the internal bubble pressure, $p_{B}$, couples the Keller-Miksis equation [Eq. (1)] to the energy equation for air inside the microbubble:

$$
\begin{gathered}
\dot{p}_{B}=\frac{3}{R}\left(\left.(\kappa-1) K \frac{\partial T}{\partial r}\right|_{r=R}-\kappa p_{B} \dot{R}\right), \\
\frac{\kappa}{\kappa-1} \frac{p_{B}}{T}\left[\frac{\partial T}{\partial t}+\frac{1}{\kappa p_{B}}\left((\kappa-1) K \frac{\partial T}{\partial r}-\frac{r \dot{p}_{B}}{3}\right) \frac{\partial T}{\partial r}\right] \\
=\dot{p}_{B}+\frac{1}{r^{2}} \frac{\partial}{\partial r}\left(r^{2} K \frac{\partial T}{\partial r}\right) .
\end{gathered}
$$

In the above equations, $T(r, t)$ is the temperature field of air inside the microbubble. Air in the bubble is treated as an ideal gas with a ratio of specific heats $\kappa$, and its thermal conductivity is given by $K=K_{A} T+K_{B}$, with empirical constants $K_{A}$ and $K_{B}[53]$ listed in Table II.

The energy equation outside the bubble is given by

$$
\frac{\partial T_{M}}{\partial t}+\frac{R^{2} \dot{R}}{r^{2}} \frac{\partial T_{M}}{\partial r}=D_{M} \nabla^{2} T_{M}+\frac{12 \mu}{\rho_{\infty} C_{p}}\left(\frac{R^{2} \dot{R}}{r^{3}}\right)^{2},
$$

where $T_{M}(r, t)$ is the temperature field in the surrounding medium. Equation (6) uses the specific heat, $C_{p}$, thermal diffusivity, $D_{M}=K_{M} /\left(\rho_{\infty} C_{p}\right)$, and thermal conductivity, $K_{M}$, of water. The final term on the right side of Eq. (6) represents the dissipation due to viscous stresses.

Boundary conditions are prescribed for the center of the bubble and far from the bubble: $\nabla T=0$ at $r=0$ and $T_{M} \rightarrow T_{\infty}$ as $r \rightarrow L$, where $T_{\infty}$ is the ambient temperature of the medium and $L \gg R$ is the arbitrarily-large outerboundary of the domain. Boundary conditions at the bubblematerial interface relate the internal bubble temperature to the temperature field in the surrounding medium, $T_{M}(r, t)$ : $\left.T\right|_{r=R}=\left.T_{M}\right|_{r=R}$ and $\left.K_{r=R} \frac{\partial T}{\partial r}\right|_{r=R}=\left.K_{M} \frac{\partial T_{M}}{\partial r}\right|_{r=R}$. Finally, summarizing the initial conditions, $R(t=0)=R_{0}, p_{B}(t=0)=$ $p_{\infty}, T(r, t)=T_{\infty}$, and $T_{M}(r, t)=T_{\infty}$. A more detailed treatment of the derivation and numerical implementation of this model can be found in Ref. [55].

\section{RESULTS}

The multiflash imaging technique allowed for clear differentiation of bubbles at different time points within the same exposure, effectively allowing higher frame-rate imaging of the bubbles, particularly in the high velocity regimes associated with growth and collapse. The utility of this imaging technique was more limited in the lower velocity regimes near the maximum radius, where the change in radius of a bubble between consecutive flashes is smaller. A series of images captured using this technique, showing the growth and first collapse of single bubbles generated via laser and acoustic nucleation in water, $0.3 \%$, and $2.5 \%$ agarose gels, are shown in Fig. 3. Note, the bright regions in the centers of laser-nucleated bubbles in the first frames are light emissions generated by the optical breakdown process responsible for nucleation.

The maximum radii of both acoustically and lasernucleated bubbles are observed to be the largest in water and to decrease monotonically in the gels as a function of increasing stiffness (Fig. 4). However, while bubbles nucleated in water by both mechanisms are larger than those nucleated in the gels, a clear nucleation-mechanism-dependent difference in the relative decrease in maximum radius is observed. That is to say, the maximum radii of laser-nucleated bubbles decreases by $15 \%$ in going from water to $0.3 \%$ gel, compared to a $3 \%$ decrease for bubbles nucleated acoustically. While more data are required to characterize the governing decay behaviors, the maximum radii of the bubbles nucleated in gels appear to decrease in an approximately power-law fashion with increasing gel stiffness. 

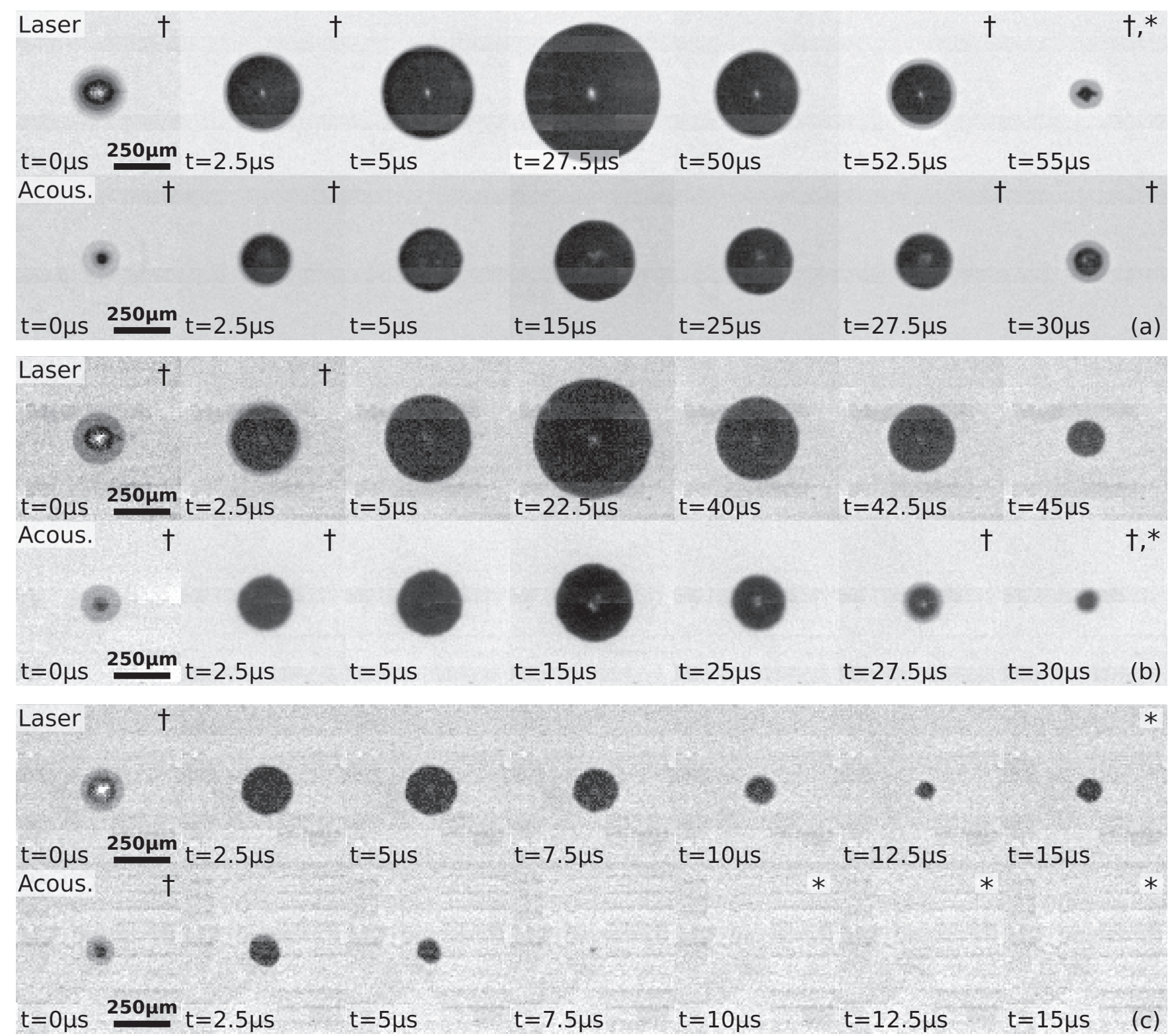

FIG. 3. Example images showing single bubbles generated using laser and acoustic nucleation mechanisms in: (a) water, (b) $0.3 \%$ agarose gel $(E=1.13 \mathrm{kPa})$, and (c) $2.5 \%$ agarose gel $(E=242 \mathrm{kPa})$. Asterisks mark frames outside of the bubbles primary lifetime which were not included in the analysis of the data. Crosses mark frames containing multiple flashes from the light source. The horizontal stripes observed in images are camera artifacts.

Although the laser-nucleated bubbles are observed to be larger than the acoustically nucleated bubbles in all media, this is not believed to be intrinsically related to the mode of nucleation. Instead, this is likely a consequence of the fixed acoustic frequency of the transducer and the fixed focal profile of the laser, which could not be changed during these experiments and which imposed restrictions on the amount of energy that could be delivered to the focus without generating additional bubbles in the field, as described in Sec. II A. As a result, the maximum radii of the bubbles could not be controlled or adjusted in this study, however, modifications to the laser beam profile or acoustic frequency are expected in general to allow the maximum radii to be controlled. It should also be pointed out here that the inability to control the maximum radii of the generated bubbles prevented the acquisition of meaningful data for the acoustically nucleated bubbles in the 5\% gel which, although they could be generated, had lifespans shorter than the duration of two camera frames $(\lesssim 5 \mu \mathrm{s})$. Hence, their dynamics and maximum radii could not be accurately assessed.

While the rebound dynamics of bubbles was not within the scope of the present study, an important nucleationmechanism-dependent difference in the decay lifetimes of the bubbles nucleated in the gels was observed. That is to say, although the acoustically nucleated bubbles were generally observed to rebound following their first collapse, they were typically seen to dissolve after $\lesssim 3$ rebounds (typically $\lesssim 35 \mu$ s). In contrast, remnant nuclei of the laser-nucleated bubbles were observed to persist in the field for up to several seconds following collapse. Evidence to this effect may be 


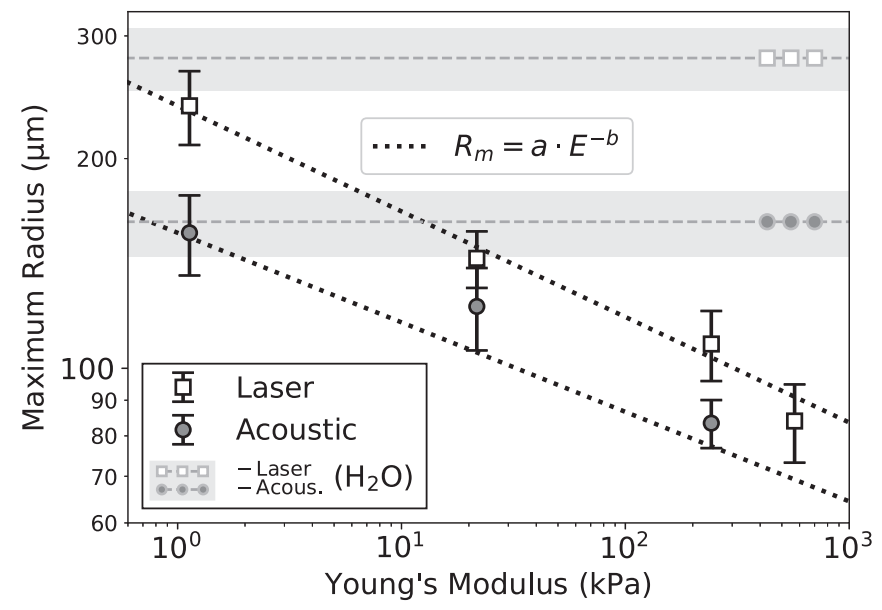

FIG. 4. Mean and standard deviations of bubble maximum radii vs. gel stiffness. The horizontal gray bars show the standard deviations of the maximum radii of the bubbles nucleated in water. The number of samples associated with each data point are shown in Fig. 5.

seen in Fig. 3(c), where, in a particularly dramatic example, no remnant gas nucleus is seen to remain following the collapse of the acoustically nucleated bubble in the $2.5 \%$ gel.

To assess for dynamical differences between bubbles, the experimentally measured radius versus time curves are compared (Fig. 5) to those predicted for the collapse of a void in an inviscid fluid by Rayleigh [1], as well as to predictions of the bubble dynamics following the viscoelastic model described in Sec. II B, using the previously reported stiffnesses of agarose (Table I) and the viscosities of water and agarose gel (Table II). Note that in the plots, the Rayleigh collapse is reflected symmetrically in time about the bubble maximum radius, reflective of the reversible nature of the Rayleigh problem (in the absence viscous/viscoelastic effects, heat transfer and so on). The dynamics of acoustically and laser-nucleated bubbles are similar to each other and similarly affected by each nucleation medium. In water and the $1.13 \mathrm{kPa}$ stiffness gels, the growths and collapses closely follow predictions for the Rayleigh cavity, but as the stiffness of the gels is increased, growth and collapse of the generated bubbles become more rapid and increasingly asymmetric, with growth being faster than collapse with respect to time. Although the early growth of the laser-nucleated bubbles $(R \lesssim 50 \mu \mathrm{m})$ could not be directly measured due to the saturation of the camera's CCD by the laser plasma-despite the camera's electronic shutter being "closed" during nucleation-these curves nevertheless reveal that the normalized growth durations of the lasernucleated bubbles are slightly larger than the acoustically nucleated bubbles in water and the $1.13 \mathrm{kPa}$ stiffness gel, but are approximately equivalent in the higher stiffness gels. No such nucleation-mechanism-dependent differences in the normalized collapse durations are observed.

Numerical simulations of the dynamics of the acoustically nucleated bubbles, using material properties previously reported in the literature and the measured acoustic pressures as inputs, are in general agreement with experimental measurements. The simulations are qualitatively accurate in predicting

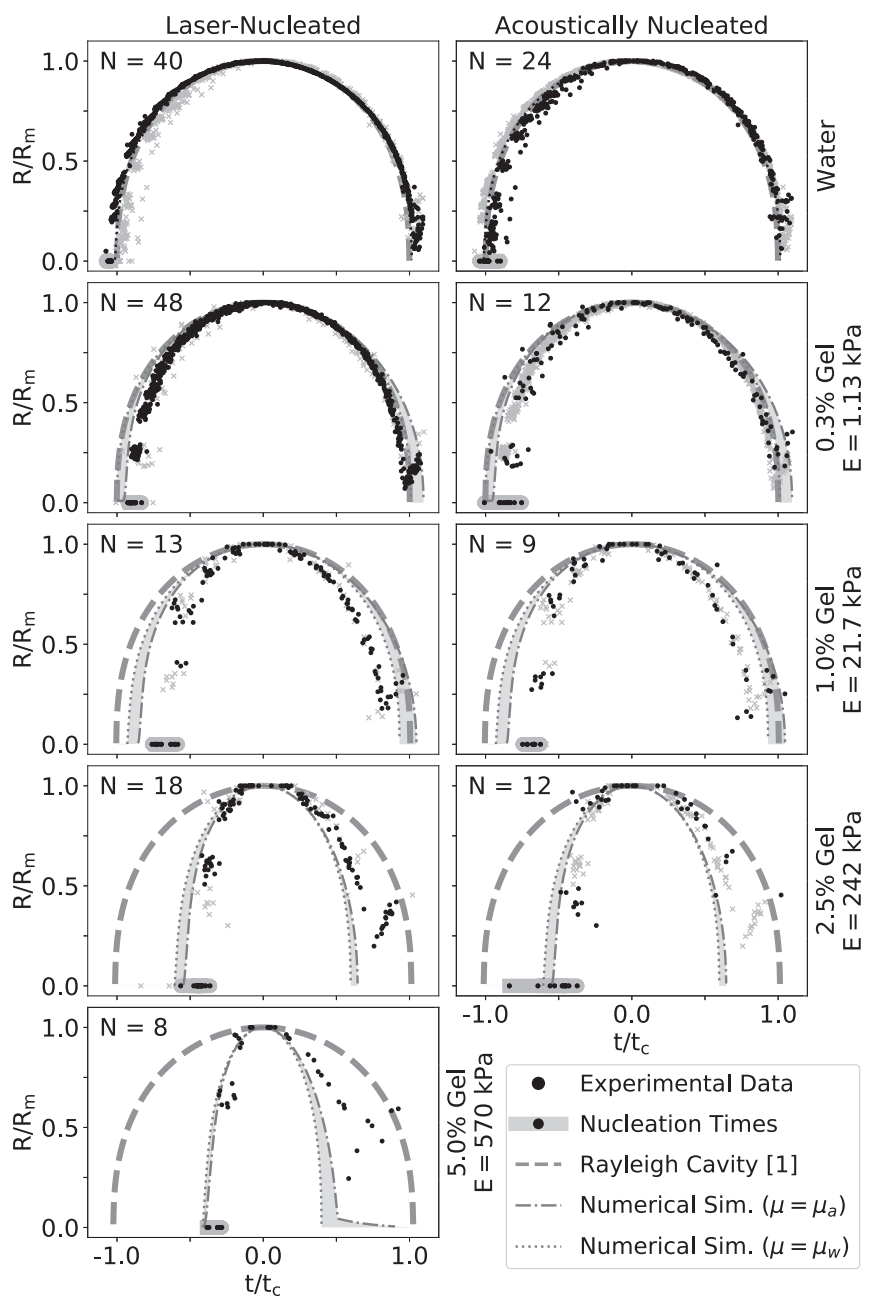

FIG. 5. Normalized $R(t)$ curves of single bubbles nucleated in water and agarose gels via laser and acoustic mechanisms. The measured values of $R_{m}$ were used to individually normalize the experimental $R(t)$ curves. $t_{c}$ is the cavity collapse time in a fluid, given by $t_{c}=0.9148 R_{m} \sqrt{\rho / P_{\infty}}$. The experimentally measured $R(t)$ curves were temporally aligned with respect to their collapses. All adjustments were bounded to within the temporal resolution error of the image containing $R_{m}$. The unlabeled gray "x"s in the plots show the measured $R(t)$ curves from the plots in the opposite column, i.e., in the "Laser-Nucleated" column the "x"s show the data from the plots in the "Acoustically Nucleated" column and vice versa. The value, $N$, in each plot corresponds to the number of cavitation events in each sample set that were measured to meet the inclusion criteria for this study. Note also that all radii captured in the image frame associated with collapse were included as data points in these plots. As such, the rising "tails" observed at later times, though made up of points captured in the frame associated with collapse, may represent the earliest portion of the first rebound.

that the growth and collapse times of the bubbles would decrease as a function of increasing gel stiffness and that the asymmetry between growth and collapse would be larger at a higher viscosity. Bubble growth is better predicted by simulations than collapse, where the normalized growth durations tend to be slightly, but uniformly, overestimated while the predictions of the collapse durations go from being over- to underestimated as a function of increasing gel stiffness. 


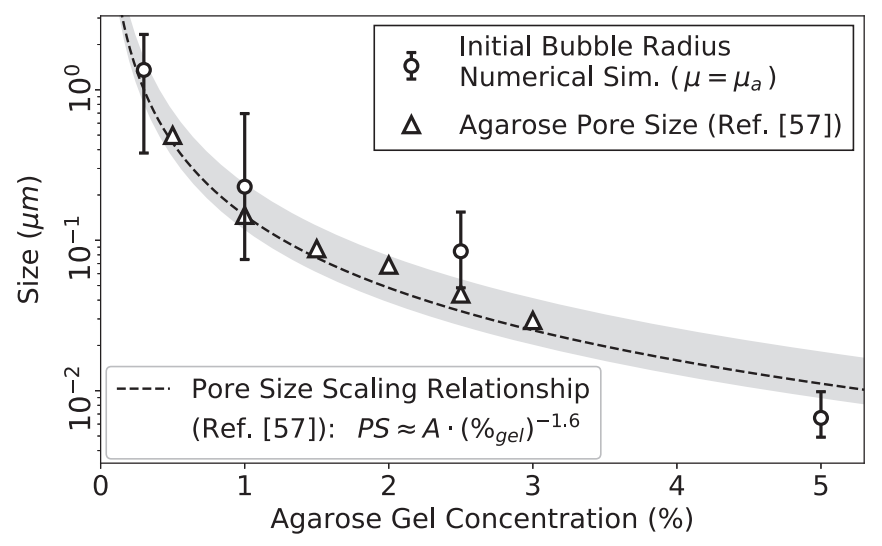

FIG. 6. Initial radii of bubbles returned from simulations and the previously reported pore sizes of agarose as a function of agarose gel concentration. Simulation results show the initial radii in simulations which produced the best agreement with the measured values $\bar{R}_{m} \pm \operatorname{std}\left(R_{m}\right)$ at each gel concentration. The data points and scaling relationship curve from Ref. [57] show the pore sizes of agarose gels in that study which were prepared following procedures in closest agreement with those in the present study. The shaded region shows the bounds of the scaling relationship in Ref. [57] for gels which were allowed to solidify at temperatures from $15^{\circ} \mathrm{C}$ to $35^{\circ} \mathrm{C}$.

The values of the initial radii found in simulations using the prescribed viscosity of agarose, $\mu=\mu_{a}$, are in good agreement with the reported pore sizes of agarose gels and follow the same approximate scaling relationship as the pore size as a function of gel concentration [57] (Fig. 6). In simulations using the viscosity of water, the initial radii are found to be significantly more uniform with values of $2.51 \mathrm{~nm}$ in water, $4.24 \mathrm{~nm}$ in the $0.3 \%$ concentration gel, and $2.33 \mathrm{~nm}$ in all $\geqslant 1 \%$ concentration gels.

\section{DISCUSSION}

In this study it was observed that the dynamics of acoustically and laser-nucleated bubbles in water and agarose gels were generally similar. The collapse of the normalized $R(t)$ curves suggests that all bubbles in each medium are governed by the same underlying physics. In the gels, the maximum radii of bubbles nucleated by both mechanisms were observed to decrease monotonically with respect to increasing gel stiffness (Fig. 4). The normalized growths and collapses of bubbles were also observed to occur faster and to become increasingly asymmetric as gel stiffness increased (Fig. 5).

The data were also suggestive of some differences associated with nucleation mechanism, particularly in the transition region from nucleation in water to nucleation in the lower stiffness/concentration gels. The maximum radii of the lasernucleated bubbles were observed to decrease faster than those of the acoustically nucleated bubbles in this region, and the normalized growth times of the laser-nucleated bubbles were observed to be longer than those of the acoustically nucleated bubbles. No such differences were apparent in the normalized collapse times or in the higher stiffness/concentration gels.

One potential explanation for the differences in the $R_{m}$ behavior and normalized growth rates in this region is that laser nucleation in the gels might be primarily due to the ionization of water trapped within the gels' pores as opposed to the ionization of the gel itself, at least at the onset. Owing to differences in the ionization- and electron-loss-rates between water and agarose, which are reasonably expected to differ, it may be the case that the energy balance of ionization events in water leads to the generation of conditions more favorable to bubble growth than those in agarose. If this were the case, then bubbles nucleated in lower concentration gels would have greater potential for growth than those nucleated in higher concentration gels, independent of the gel's viscoelastic properties, owing to the larger water-containing pores within. As the pore sizes in the agarose gels in this study varied by up to three orders of magnitude between gel concentrations, ranging from approximately $1.4 \mu \mathrm{m}$ at a concentration of $0.3 \%$ to $\sim 8 \mathrm{~nm}$ at a concentration of $5 \%$ (Fig. 6), this could have resulted in perceptible differences in the dynamics of the bubbles nucleated in each medium.

Macro-scale differences in the drivers of bubble growth should also be considered, particularly in relation to the slower normalized growth rates of the laser-nucleated bubbles compared to the acoustically nucleated ones in water and the $0.3 \%$ gel. Laser-nucleated bubbles grow as the result of a nearly instantaneous buildup of internal positive pressure at the ionization site, whereas the growth of acoustically nucleated bubbles follows the application of an external negative pressure at the acoustic focus. The near-vacuum internal conditions generated within the bubbles during acoustic nucleation are expected to remain consistent between all nucleation media. The vapor and gas byproducts generated by the ionization event during laser-nucleation, however, as well as the thermodynamic conditions that develop within the bubbles thereafter, are expected to vary as a function of nucleation medium. If ionization events in water generate internal conditions that are more supportive of bubble growth than the near-vacuum conditions generated during acoustic nucleation, then the normalized growth durations of the laser-nucleated bubbles would be extended.

Evidence to the effect of differing internal conditions was noted in Fig. 3(c), where a remnant gas nucleus was observed to remain following the collapse of the laser-nucleated bubble but not the acoustically nucleated one in the $2.5 \%$ gel. That the acoustically nucleated bubbles decay so quickly without rebounding significantly may have important consequences for modeling their dynamics in viscoelastic media as the rebound and decay of bubbles to stable equilibria are important features for determining model parameters including the initial radius, $R_{0}$, which was unknown in the present study.

Simulations were in reasonable overall agreement with experiments and were qualitatively accurate in their predictions of the bubble dynamics observed. However, the simulations were quantitatively inaccurate in predicting the magnitudes of the changes in the normalized dynamics observed experimentally. As significantly better quantitative agreement with experimental observations could be achieved by arbitrarily adjusting the stiffnesses and viscosities of the gels in simulations, these results may indicate that the reported values of these properties [23,25,49] in agarose are inaccurate, or, more specifically, that the values of these properties measured 
under quasi-static conditions do not accurately reflect those at the high to ultra-high strain-rates generated by cavitation $\left(10^{3}-10^{8} \mathrm{~s}^{-1}\right)[15,27,33]$.

The assumption of material homogeneity in the model is likely also not valid for agarose gels, particularly in the early parts of a bubble's lifetime. The first expansion of a bubble can only occur into the undisturbed, inhomogeneous agarose gel and thus any influence of material inhomogeneities on the resulting dynamics would be most pronounced during this phase of the bubble's evolution. For example, if the expansion of the bubble irreversibly disrupted the structure of the surrounding gel, then the material into which the bubble grew would not be equivalent to that into which it subsequently collapsed. This could result in unequal forcing on the bubble by its surroundings during each phase of its evolution and could contribute to the asymmetry between growth and collapse observed experimentally. Phenomena analogous to this, wherein the mechanical response of a material during the first loading is different than that during subsequent unloading and loading cycles due to structural changes in the material, have been described previously (e.g., the Mullins effect in rubbers [58]), but would not have been captured in the present simulations. This might also help explain the observation that the simulations tended to be more consistent in their predictions of bubble growth than collapse. As the previously measured elasticities and viscosities of agarose were measured in undisturbed gel samples, simulations of the dynamics using these values would presumably be most accurate during bubble growth while the gel is still in an undisturbed state.

The agreement between the initial radii of the bubbles found in simulations and the previously reported pore sizes of agarose gel as a function of the gel concentration [57] also suggests that the nucleation dynamics and growth of the acoustically nucleated bubbles are influenced by agarose's pores. This result may indicate, for example, that nucleation is initiated in the water within the gel's pores but, upon growing to fill the pore volume, the dynamics of the bubbles are altered in such a way that they then behave as though they were "nucleated" from pore-sized initial radii. Owing to the resolution limits in images during experiments, estimates of the initial radii in simulations could only be made based on comparison to measurements from this latter phase of a bubble's growth. Additionally, as the time required for the bubbles to grow to the size of the pores in all cases would have been $\leqslant 0.1 \%$ of the acoustic period, the acoustic environments experienced by the pore-sized bubbles during 'nucleation' would be effectively equivalent to those experienced by the nuclei in the water.

Perhaps the biggest limitation of this study was the inability to control the maximum radii of the generated bubbles. This prevented direct comparison between the dynamics of equivalently sized bubbles generated by both nucleation mechanisms and in all nucleation media, and therefore, any potential size-dependent effects on the observed dynamics could not be assessed. Combined with the temporal and spatial resolution limits in images, this also accounts for the absence of usable data for the acoustically nucleated bubbles in the $5 \%$ gel, which were too small and short-lived to accurately measure and thus were not included in this report.
Reliably nucleating single, spherical bubbles and adequately illuminating bubbles nucleated in gels while achieving the desired temporal and spatial resolutions also proved challenging during this study. In particular, the probability of nucleating single, spherical bubbles during each nucleation attempt in the gels was only $15 \%-20 \%$, which resulted in fewer of these bubbles falling within the inclusion criteria for this study. Even among those that did meet the inclusion criteria, small amplitude shape instabilities were often observed [e.g., the acoustically nucleated bubble in Fig. 3(b)], which may have contributed to the noise apparent in the $R(t)$ curves. While increasing gel concentrations did not appreciably affect the proportion of bubbles falling outside the inclusion criteria, or the apparent generation of the evident shape instabilities, it did impose limits on the temporal resolution achievable using the multiflash imaging technique as higher concentration gels were significantly less translucent to the blue LED backlight, which required using more sparsely distributed, longer duration flashes to achieve adequate illumination. This may have limited the ability to resolve some instabilities and also accounts for the discretization of points in the $R(t)$ curves in higher concentration gels. Lighting also limited the spatial resolution of images, as a higher magnification would have come at the cost of reducing the amount of light reaching the camera sensor. This may have limited our ability to resolve asymmetries in the smaller bubbles and likely accounts for the noisier $R(t)$ data in Fig. 5 for the bubbles nucleated in the $\geqslant 21.7 \mathrm{kPa}$ stiffness gels.

\section{CONCLUSION}

In this study, we observed that the dynamics of acoustically and laser-nucleated bubbles are generally similar in water and agarose gels, and that both the stiffness and viscosity, and to a lesser extent the nucleation method, can impact bubble dynamics. Increasing gel stiffness suppressed maximum bubble growth and increased the rate at which the bubbles collapsed. Asymmetries in the growths and collapses of the bubbles were observed for bubbles nucleated in gels but not in water, which simulations indicate is likely due, at least in part, to the higher viscosity of agarose compared to water.

Differences in the maximum radii and normalized growth rates with respect to nucleation mechanism and gel stiffness were observed and are believed to be due to differences in the ionization processes in water and agarose gels as well as macro-scale differences in the drivers of bubble growth. Observations of stable gas nuclei in the gels following laser nucleation but not acoustic nucleation are indicative of differences in the compositions and thermodynamic environments generated within bubbles nucleated by each mechanism. The rapid dissolution of acoustically nucleated bubbles, without decaying to stable equilibrium radii, could have important implications for modeling their behavior.

The initial radii of the bubbles predicted in numerical simulations using the viscosity of agarose gel were in good agreement with previously reported values of the pore sizes of agarose as a function of gel concentration, and may indicate that the pores have an impact on the nucleation and growth dynamics. However, while simulations were qualitatively accurate in capturing the shortening of the growth and collapse 
times with increasing gel stiffness and the asymmetry between them at the higher viscosity, the predictions of the normalized dynamics on the whole were not quantitatively accurate. It is believed that this is likely a result of the assumption of material homogeneity in the model being violated during the first growth-collapse cycle of the bubbles' evolutions, as well as the low strain-rate stiffness and viscosity values not holding at the high strain-rates generated during cavitation, for example due to unaccounted-for effects such as strain/strainrate stiffening.

The observations presented in this study provided useful insights about the effects of the viscoelastic properties on the resulting bubble dynamics, and suggest that the dynamics of laser-nucleated bubbles in agarose gel are generally representative of those nucleated acoustically. Potentially important nucleation-mechanism-dependent differences in dynamics were observed, however, which suggest this may not be true in all media, particularly in inhomogeneous or porous media where localized differences in the ionization dynamics could result in conditions more or less favorable to bubble growth, independent of a material's viscoelastic properties. While laser nucleation allows for easier study of controlled cavitation activity, the disagreement between the normalized growths of the acoustically and laser-nucleated bubbles as a function of nucleation medium is suggestive of limits to using laser-nucleated bubbles to inform models of acoustically nucleated bubbles, especially during the first growth-collapse cycle where nucleation-mechanism-dependent effects are likely to have the greatest impacts on dynamics.

\section{ACKNOWLEDGMENTS}

The authors thank Alice Lux Fawzi for her help in organizing this project. This work supported by ONR Grant No. N00014-17-1-2058 (under Dr. T. Bentley) and the Focused Ultrasound Foundation Grant No. 24332.
[1] L. Rayleigh, On the pressure developed in a liquid during the collapse of a spherical cavity, Philos. Mag. 34, 94 (1917).

[2] W. Lauterborn and H. Bolle, Experimental investigations of cavitation-bubble collapse in the neighbourhood of a solid boundary, J. Fluid Mech. 72, 391 (1975).

[3] K. Suslick, S. Doktycz, and E. Flint, On the origin of sonoluminescence and sonochemistry, Ultrasonics 28, 280 (1990).

[4] D. F. Gaitan, L. A. Crum, C. C. Church, and R. A. Roy, Sonoluminescence and bubble dynamics for a single, stable, cavitation bubble, J. Acoust. Soc. Am. 91, 3166 (1992).

[5] R. G. Holt and R. Roy, Measurements of bubble-enhanced heating from focused, MHz-frequency ultrasound in a tissuemimicking material, Ultrasound Med. Biol. 27, 1399 (2001).

[6] A. K. Khachatryan, S. Aloyan, P. May, R. Sargsyan, V. Khachatryan, and V. Baghdasaryan, Graphite-to-diamond transformation induced by ultrasound cavitation, Diam. Relat. Mater. 17, 931 (2008).

[7] J. R. Sukovich, A. Sampathkumar, P. A. Anderson, R. G. Holt, Y. A. Pishchalnikov, and D. F. Gaitan, Temporally and spatially resolved imaging of laser-nucleated bubble cloud sonoluminescence, Phys. Rev. E 85, 056605 (2012).

[8] A. D. Maxwell, C. A. Cain, T. L. Hall, J. B. Fowlkes, and Z. Xu, Probability of cavitation for single ultrasound pulses applied to tissues and tissue-mimicking materials, Ultrasound Med. Biol. 39, 449 (2013).

[9] J. R. Sukovich, P. A. Anderson, A. Sampathkumar, D. F. Gaitan, Y. A. Pishchalnikov, and R. G. Holt, Outcomes of the collapse of a large bubble in water at high ambient pressures, Phys. Rev. E 95, 043101 (2017).

[10] M. Kornfeld and L. Suvorov, On the destructive action of cavitation, J. Appl. Phys. 15, 495 (1944).

[11] R. K. Bhaskaracharya, S. Kentish, and M. Ashokkumar, Selected applications of ultrasonics in food processing, Food Eng. Rev. 1, 31 (2009).

[12] K. E. Hitchcock and C. K. Holland, Ultrasound-assisted thrombolysis for stroke therapy better thrombus break-up with bubbles, Stroke 41, S50 (2010).

[13] J. E. Parsons, C. A. Cain, G. D. Abrams, and J. B. Fowlkes, Pulsed cavitational ultrasound therapy for controlled tissue homogenization, Ultrasound Med. Biol. 32, 115 (2006).

[14] J. Goeller, A. Wardlaw, D. Treichler, J. O'Bruba, and G. Weiss, Investigation of cavitation as a possible damage mechanism in blast-induced traumatic brain injury, J. Neurotrauma 29, 1970 (2012).

[15] E. Bar-Kochba, M. T. Scimone, J. B. Estrada, and C. Franck, Strain and rate-dependent neuronal injury in a $3 \mathrm{D}$ in vitro compression model of traumatic brain injury, Sci. Rep. 6, 30550 (2016).

[16] T. L. Hall, K. Kieran, K. Ives, J. B. Fowlkes, C. A. Cain, and W. W. Roberts, Histotripsy of rabbit renal tissue in vivo: Temporal histologic trends, J. Endourol. 21, 1159 (2007).

[17] Z. Xu, G. Owens, D. Gordon, C. Cain, and A. Ludomirsky, Noninvasive creation of an atrial septal defect by histotripsy in a canine model, Circulation 121, 742 (2010).

[18] J. C. Wheat, T. L. Hall, C. R. Hempel, C. A. Cain, Z. Xu, and W. W. Roberts, Prostate histotripsy in an anticoagulated model, Urology 75, 207 (2010).

[19] E. Vlaisavljevich, Y. Kim, S. Allen, G. Owens, S. Pelletier, C. Cain, K. Ives, and Z. Xu, Image-guided non-invasive ultrasound liver ablation using histotripsy: Feasibility study in an in vivo porcine model, Ultrasound Med. Biol. 39, 1398 (2013).

[20] X. Zhang, G. E. Owens, C. A. Cain, H. S. Gurm, J. Macoskey, and $\mathrm{Z}$. $\mathrm{Xu}$, Histotripsy thrombolysis on retracted clots, Ultrasound Med. Biol. 42, 1903 (2016).

[21] J. R. Sukovich, C. A. Cain, A. S. Pandey, N. Chaudhary, S. Camelo-Piragua, S. P. Allen, T. L. Hall, J. Snell, Z. Xu, J. M. Cannata, D. Teofilovic, J. A. Bertolina, N. Kassell, and Z. $\mathrm{Xu}$, In vivo histotripsy brain treatment, J. Neurosurg. (2018), doi:10.3171/2018.4.JNS172652.

[22] V. A. Khokhlova, J. B. Fowlkes, W. W. Roberts, G. R. Schade, Z. Xu, T. D. Khokhlova, T. L. Hall, A. D. Maxwell, Y.-N. Wang, and C. A. Cain, Histotripsy methods in mechanical disintegration of tissue: Towards clinical applications, Int. J. Hypertherm. 31, 145 (2015).

[23] P. Movahed, W. Kreider, A. D. Maxwell, S. B. Hutchens, and J. B. Freund, Cavitation-induced damage of soft materials by 
focused ultrasound bursts: A fracture-based bubble dynamics model, J. Acoust. Soc. Am. 140, 1374 (2016).

[24] E. Vlaisavljevich, C. A. Cain, and Z. Xu, The effect of histotripsy on tissues with different mechanical properties, in Proceedings of the Ultrasonics Symposium (IUS'11) (IEEE, New York, NY, 2011), pp. 1490-1493.

[25] E. Vlaisavljevich, K.-W. Lin, A. Maxwell, M. T. Warnez, L. Mancia, R. Singh, A. J. Putnam, B. Fowlkes, E. Johnsen, C. Cain et al., Effects of ultrasound frequency and tissue stiffness on the histotripsy intrinsic threshold for cavitation, Ultrasound Med. Biol. 41, 1651 (2015).

[26] E. Vlaisavljevich, A. Maxwell, M. Warnez, E. Johnsen, C. Cain, and Z. Xu, Histotripsy-induced cavitation cloud initiation thresholds in tissues of different mechanical properties, IEEE Trans. Ultrason. Ferroelectr. Freq. Control 61, 341 (2014).

[27] J. B. Estrada, C. Barajas, D. L. Henann, E. Johnsen, and C. Franck, High strain-rate soft material characterization via inertial cavitation, J. Mech. Phys. Solids 112, 291 (2018).

[28] X. Yang and C. C. Church, A model for the dynamics of gas bubbles in soft tissue, J. Acoust. Soc. Am. 118, 3595 (2005).

[29] C. Hua and E. Johnsen, Nonlinear oscillations following the rayleigh collapse of a gas bubble in a linear viscoelastic (tissuelike) medium, Phys. Fluids 25, 083101 (2013).

[30] R. Gaudron, M. Warnez, and E. Johnsen, Bubble dynamics in a viscoelastic medium with nonlinear elasticity, J. Fluid Mech. 766, 54 (2015).

[31] M. Warnez and E. Johnsen, Numerical modeling of bubble dynamics in viscoelastic media with relaxation, Phys. Fluids 27, 063103 (2015).

[32] F. Hamaguchi and K. Ando, Linear oscillation of gas bubbles in a viscoelastic material under ultrasound irradiation, Phys. Fluids 27, 113103 (2015).

[33] D. K. Cullen, C. M. Simon, and M. C. LaPlaca, Strain ratedependent induction of reactive astrogliosis and cell death in three-dimensional neuronal-astrocytic co-cultures, Brain Res. 1158, 103 (2007).

[34] P. Huber, J. Debus, K. Jöchle, I. Simiantonakis, J. Jenne, R. Rastert, J. Spoo, W. J. Lorenz, and M. Wannenmacher, Control of cavitation activity by different shockwave pulsing regimes, Phys. Med. Biol. 44, 1427 (1999).

[35] Y. Hong, M. Sarntinoranont, G. Subhash, S. Canchi, and M. King, Localized tissue surrogate deformation due to controlled single bubble cavitation, Exper. Mech. 56, 97 (2016).

[36] A. Vogel, M. Capon, M. N. Asiyo-Vogel, and R. Birngruber, Intraocular photodisruption with picosecond and nanosecond laser pulses: Tissue effects in cornea, lens, and retina, Invest. Ophthalmol. Visual Sci. 35, 3032 (1994).

[37] I. Akhatov, O. Lindau, A. Topolnikov, R. Mettin, N. Vakhitova, and W. Lauterborn, Collapse and rebound of a laser-induced cavitation bubble, Phys. Fluids 13, 2805 (2001).

[38] T. Kurz, D. Kröninger, R. Geisler, and W. Lauterborn, Optic cavitation in an ultrasonic field, Phys. Rev. E 74, 066307 (2006).

[39] A. V. Cherian and K. R. Rau, Pulsed-laser-induced damage in rat corneas: Time-resolved imaging of physical effects and acute biological response, J. Biomed. Opt. 13, 024009 (2008).

[40] B. D. Storey and A. J. Szeri, Water vapour, sonoluminescence and sonochemistry, in Proceedings of the Royal Society of London A: Mathematical, Physical and Engineering Sciences, Vol. 456 (The Royal Society, London, 2000), pp. 1685-1709.
[41] M. Margulis and I. Margulis, Luminescence mechanism of acoustic and laser-induced cavitation, Acoust. Phys. 52, 283 (2006).

[42] P. K. Kennedy, A first-order model for computation of laserindunced breakdown thresholds in ocular and aqueous media: Part I-Theory, IEEE J. Quant. Electron. 31, 2241 (1995).

[43] A. Vogel, K. Nahen, D. Theisen, and J. Noack, Plasma formation in water by picosecond and nanosecond Nd:YAG laser pulses-Part I: Optical breakdown at threshold and super threshold irradiance, IEEE J. Sel. Top. Quantum Electron. 2, 847 (1996).

[44] A. Vogel, S. Busch, and U. Parlitz, Shock wave emission and cavitation bubble generation by picosecond and nanosecond optical breakdown in water, J. Acoust. Soc. Am. 100, 148 (1996).

[45] P. Aymard, D. R. Martin, K. Plucknett, T. J. Foster, A. H. Clark, and I. T. Norton, Influence of thermal history on the structural and mechanical properties of agarose gels, Biopolymers: Orig. Res. Biomol. 59, 131 (2001).

[46] E. Vlaisavljevich, Z. Xu, A. D. Maxwell, L. Mancia, X. Zhang, K.-W. Lin, A. P. Duryea, J. R. Sukovich, T. L. Hall, E. Johnsen et al., Effects of temperature on the histotripsy intrinsic threshold for cavitation, IEEE Trans. Ultrason. Ferroelectr. Freq. Control 63, 1064 (2016).

[47] E. Vlaisavljevich, A. Maxwell, L. Mancia, E. Johnsen, C. Cain, and $\mathrm{Z}$. Xu, Visualizing the histotripsy process: Bubble cloudcancer cell interactions in a tissue-mimicking environment, Ultrasound Med. Biol. 42, 2466 (2016).

[48] L. Mancia, E. Vlaisavljevich, Z. Xu, and E. Johnsen, Predicting tissue susceptibility to mechanical cavitation damage in therapeutic ultrasound, Ultrasound Med. Biol. 43, 1421 (2017).

[49] H. Panda, The Complete Book on Gums and Stabilizers for Food Industry (Asia Pacific Business Press, New Delhi, 2010).

[50] I. R. Webb, S. J. Payne, and C.-C. Coussios, The effect of temperature and viscoelasticity on cavitation dynamics during ultrasonic ablation, J. Acoust. Soc. Am. 130, 3458 (2011).

[51] E. Vlaisavljevich, K.-W. Lin, M. T. Warnez, R. Singh, L. Mancia, A. J. Putnam, E. Johnsen, C. Cain, and Z. Xu, Effects of tissue stiffness, ultrasound frequency, and pressure on histotripsy-induced cavitation bubble behavior, Phys. Med. Biol. 60, 2271 (2015).

[52] J. B. Keller and M. Miksis, Bubble oscillations of large amplitude, J. Acoust. Soc. Am. 68, 628 (1980).

[53] A. Prosperetti, L. A. Crum, and K. W. Commander, Nonlinear bubble dynamics, J. Acoust. Soc. Am. 83, 502 (1988).

[54] V. Kamath, A. Prosperetti, and F. Egolfopoulos, A theoretical study of sonoluminescence, J. Acoust. Soc. Am. 94, 248 (1993).

[55] C. Barajas and E. Johnsen, The effects of heat and mass diffusion on freely oscillating bubbles in a viscoelastic, tissue-like medium, J. Acoust. Soc. Am. 141, 908 (2017).

[56] K. B. Bader and V. Bollen, The influence of gas diffusion on bubble persistence in shock-scattering histotripsy, J. Acoust. Soc. Am. 143, EL481 (2018).

[57] J. Narayanan, J.-Y. Xiong, and X.-Y. Liu, Determination of agarose gel pore size: Absorbance measurements vis a vis other techniques, in Proceedings of the Journal of Physics Conference Series, Vol. 28 (IOP Publishing, Philadelphia, PA, 2006), p. 83.

[58] J. Diani, B. Fayolle, and P. Gilormini, A review on the Mullins effect, Eur. Polymer J. 45, 601 (2009). 